\title{
CUSTO-CONSUMO DE BEBIDAS ALCOÓLICAS ENTRE HOMENS E MULHERES EM UMA REGIÃO DA AMAZÔNIA LEGAL
}

\author{
COST-CONSUMPTION OF ALCOHOLIC BEVERAGES AMONG MEN \\ AND WOMEN IN THE BRAZIL'S LEGAL AMAZON REGION
}

\section{COSTO-CONSUMO DE BEBIDAS ALCOHÓLICAS ENTRE HOMBRES Y MUJERES EN UNA REGIÓN DE LA AMAZONIA LEGAL}

\author{
Vagner Ferreira Do Nascimento* \\ Edelson Emídio Da Silva** \\ Thalise Yuri HatTori ${ }^{* * *}$ \\ Ana Cláudia Pereira Terças-Trettel ${ }^{* * * *}$ \\ Alisséia Guimarães Lemes***** \\ Margarita Antonia Villar Luis******
}

\section{RESUMO}

Objetivo: Investigar o custo-consumo de bebidas alcoólicas entre homens e mulheres em uma regiáo da Amazônia Legal. Material e Método: Estudo exploratório e com abordagem mista, realizado em bairros de periferia de uma regiáo da Amazônia Legal, nos meses de agosto a dezembro de 2019, junto a consumidores de bebidas álcoolicas, totalizando 60 participantes. Aplicou um roteiro semiestruturado e o Alcohol Use Disorders Identification Test. Utilizou-se o discurso do sujeito coletivo, e análise de correlaçáo "Coeficiente de Contingência C”. Respeitou todos os aspectos éticos em pesquisa. Resultados: Predominou mulheres com consumo de risco e homens em dependência química. Observou-se uma forte associação do teor alcoólico $(C=0,481 ; \mathrm{p}=0,0004)$ e preço da bebida $(C=0,386 ; \mathrm{p}=0,0197)$ com todos os tipos de padráo de uso de álcool. Para todos os homens, o teor alcoólico influencia o consumo, assim como o preço das bebidas. Entre as mulheres, sendo distinto somente o teor alcoólico. Conclusão: Esses achados apontam que os aspectos que determinam o custo-consumo

\footnotetext{
*Enfermeiro. Doutor em Bioética. Universidade do Estado de Mato Grosso (UNEMAT), Tangará da Serra, Mato Grosso, Brasil. ORCID: https://orcid.org/0000-0002-3355-163X Email: vagnernascimento@unemat.br Autor correspondente

${ }^{* *}$ Enfermeiro. Graduado em Enfermagem Universidade do Estado de Mato Grosso (UNEMAT). Tangará da Serra, Mato Grosso, Brasil. ORCID: https://orcid.org/0000-0003-0650-7016 E-mail: edelson11011@gmail.com

***Enfermeira. Mestre em Ciências da Saúde. Universidade do Estado de Mato Grosso (UNEMAT). Tangará da Serra, Mato Grosso, Brasil. ORCID: https://orcid.org/0000-0003-4491-0375 E-mail: thalise@unemat.br

****Enfermeira. Doutora em Medicina Tropical. Universidade do Estado de Mato Grosso (UNEMAT). Tangará da Serra, Mato Grosso, Brasil. ORCID: https://orcid.org/0000-0001-8761-3325 E-mail: ana.claudia@unemat.br

*****Enfermeira. Doutora em Enfermagem Psiquiátrica. Universidade Federal de Mato Grosso (UFMT). Barra do Garças, Mato Grosso, Brasil. ORCID: https://orcid.org/0000-0001-6155-6473 E-mail: alisseia@hotmail.com

******Enfermeira. Departamento de Enfermagem Psiquiátrica e Ciências Humanas da Escola de Enfermagem de Ribeirão Preto da Universidade de São Paulo (EERP/USP), Ribeirão Preto, São Paulo, Brasil. ORCID: https://orcid.org/0000-0002-9907-5146 E-mail: margarit@eerp.usp.br
} 
são importantes elementos para fundamentar políticas públicas voltadas ao cuidado de pessoas em risco ou na condição de dependência de álcool.

Palavras-chave: Comportamento; Alcoolismo; Homens; Mulheres.

\begin{abstract}
Objective: To investigate the cost-consumption of alcoholic beverages among men and women in a region of the Brazil's Legal Amazon. Material and Method: Exploratory study with a mixed approach, carried out in neighborhoods on the periphery of a region of the Legal Amazon, from August to December 2019, with consumers of alcoholic beverages, totaling 60 participants. A semi-structured script and the Alcohol Use Disorders Identification Test was applied. The collective subject discourse and the correlation analysis "Contingency Coefficient C" were used. All ethical aspects of the research were respected. Results: There was a predominance of women at risk of consumption and men in chemical dependency. There was also a strong association between alcohol content $(C=0.481 ; \mathrm{p}=0.0004)$ and beverage price $(\mathrm{C}=0.386 ; \mathrm{p}=0.0197)$ with all types of alcohol consumption patterns. For all men, the alcohol content has an effect on consumption, as well as on the price of beverages. Among women, only the alcohol content varies. Conclusion: These findings point out that the aspects that determine cost-consumption are important elements to promote public policies aimed at assisting people at risk or affected by alcohol dependence.
\end{abstract}

Key words: Behavior; Alcoholism; Men; Women.

\title{
RESUMEN
}

Objetivo: Investigar el costo-consumo de bebidas alcohólicas entre hombres y mujeres en una región de la Amazonia Legal. Material y Método: Estudio exploratorio con enfoque mixto, realizado en barrios de la periferia de una región de la Amazonía Legal, de agosto a diciembre de 2019, con consumidores de bebidas alcohólicas, totalizando 60 participantes. Se aplicó un guión semiestructurado y el Alcohol Use Disorders Identification Test. Se utilizó el discurso del sujeto colectivo y el análisis de correlación "Coeficiente de contingencia C". Se respetaron todos los aspectos éticos en la investigación. Resultados: hubo predominio de mujeres en riesgo de consumo y hombres en dependencia química. Hubo una fuerte asociación del contenido de alcohol $(\mathrm{C}=0,481 ; \mathrm{p}=0,0004)$ y el precio de la bebida $(C=0,386 ; p=0,0197)$ con todos los tipos de patrones de consumo de alcohol. Para todos los hombres, el contenido de alcohol influye en el consumo, así como en el precio de las bebidas. Entre las mujeres, solo el contenido de alcohol es distinto. Conclusión: Estos hallazgos señalan que los aspectos que determinan el costo-consumo son elementos importantes para sustentar las políticas públicas dirigidas a la atención de personas en riesgo o en condición de dependencia del alcohol.

Palabras clave: Comportamiento; Alcoholismo; Hombres; Mujeres.

Data de recepção: 13/08/2020

\section{INTRODUÇÁO}

O álcool é a substância mais consumida no mundo, com maior aceitação e reconhecimento pela sociedade, e também é a que causa mais danos por riscos voluntários ${ }^{(1)}$. Mundialmente, cerca de 3,3 milhóes de todas as mortes e diversas patologias estão relacionadas à ingestão de álcool. $\mathrm{Na}$ América
Data de aceitação: 23/04/2021

Latina, essa situação é proporcionalmente mais grave, não somente pelo quantitativo de óbitos, mas pelos anos de vida saudáveis perdidos. Apesar disso, as questóes relacionadas com o fenômeno do alcoolismo seguem tendo pouca prioridade nas agendas de saúde da maioria dos países latinos, sem avaliação dos custos e danos gerados. Em contraponto, as políticas públicas existentes na 
América Latina ainda não conseguem regulamentar medidas assertivas relacionadas ao consumo, acesso e preço das bebidas alcoólicas ${ }^{(2)}$.

Pesquisadores apontam que o preço dessas substâncias nos locais em que estáo disponíveis, pode determinar o consumo. E para reduzir o uso, julgam que o valor de venda do produto deve ser aumentado ${ }^{(3)}$, no entanto, além do custo, a acessibilidade também fomenta o consumo e pode torná-lo mais intenso, com aparecimento de problemas associados ou agravamento daqueles já existentes $^{(4)}$.

O custo-consumo de bebidas alcoólicas é determinado por aspectos econômicos (preço e taxaçóes que elevam o custo), de varejo (facilidades de compra e acesso às bebidas alcoólicas) e sociais como fontes de acesso e perfil étnico-cultural da população $^{(5)}$.

Estudo realizado na Austrália aponta que bebedores mais frequentes são pouco influenciados quanto ao preço ${ }^{(6)}$. Contudo, o minimum unit prices (MUPs), ou seja, os preços unitários mínimos, política conhecida no Reino Unido desde 2012 para reduzir o consumo de álcool, mostrou-se uma medida eficaz de saúde pública e que afeta diretamente indivíduos com consumo abusivo e prejudicial $^{(7)}$.

Em relação ao gênero, o consumo de álcool entre os homens sempre se mostrou mais elevado em comparação ao da mulher, porém essa diferença vem diminuindo devido a um aumento progressivo no consumo feminino(8). Há recomendações que orientam doses distintas entre mulheres e homens, pelas particularidades fisiológicas e psicoemocionais, velocidade de intoxicaçáo, período de dependência e probabilidades de recuperação ${ }^{(9)}$. Observa-se também, uma tendência de os estudos agruparem todos os gêneros em uma mesma análise, porém isso impede que o gênero feminino, normalmente com menor busca ou busca tardia pelos serviços de tratamento da dependência química, por aspectos sociais, como autopercepção de preconceito, julgamento, estigma, culpa, vergonha e possíveis violências no contexto familiar e institucional ${ }^{(10)}$, receba a devida atenção e acolhimento integral, o que reforça a importância em dar visibilidade às características de cada gênero.

Na Amazônia Legal, o Estado de Mato Grosso possui uma das maiores taxas de mortalidade atribuída ao uso de álcool ${ }^{(11)}$. E pesquisas realizadas nessa região também apontaram a gravidade do consumo de álcool e as influências do meio e marketing no acesso, frequência e quantidade ingerida $^{(12,13)}$. Porém, ainda não se conhece, as relaçôes de custo-consumo de bebidas alcoólicas nessa população. Além disso, dada as questôes de precariedade de infraestrutura, os vazios assistenciais para atender essa população e a concepção de alguns profissionais médicos e enfermeiros da Amazônia ${ }^{(14)}$ no acolhimento e gestão do cuidado em saúde mental, especificamente nessas demandas, podem estar implicando no crescimento do consumo de álcool entre homens e mulheres, e impactando em danos em diversas esferas da vida de ambos.

Nesse panorama, tendo em vista o alcance dos impactos do álcool à pessoa tanto em caráter individual como ao meio próximo e a sociedade, são necessários novos estudos sobre o comportamento de compra e consumo ${ }^{(15)}$, especialmente na região amazônica, onde as questóes geopolíticas, como a grande distância entre as cidades, poucas estradas com boa trafegabilidade e baixo quantitativo de profissionais da saúde ${ }^{(16)}$, são entendidos como obstáculos para efetivação de intervençóes breves e efetivas, bem como cuidados mais duradouros com essa clientela. Assim, considerando a importância da temática, o estudo objetivou investigar o custoconsumo de bebidas alcoólicas entre homens e mulheres em uma regiáo da Amazônia Legal.

\section{MATERIAL E MÉTODO}

Trata-se de estudo exploratório e com abordagem mista. O estudo foi realizado em bairros de periferia do maior município de uma região da Amazônia Legal, nos meses de agosto a dezembro do ano 2019. A escolha dos bairros ocorreu com base em três critérios: população de 4.000 a 5.000 pessoas (cadastros no sistema de Atenção Básica), regióes da periferia do município e existência de supermercados de médio porte. Três bairros (A, B e C) foram os únicos da periferia do município que se encaixaram nos critérios; todos possuíam um único estabelecimento comercial e com esse perfil. A inclusão de bairros com supermercado desse porte visou atender a possibilidade de maior acesso a variedade de bebidas alcoólicas.

Foram incluídos no estudo os sujeitos que obedeceram aos critérios de inclusão: ser maior 
de 18 anos, que verbalizassem o consumo de algum tipo de bebida alcoólica, independente da frequência e quantidade ingerida. Foram excluídos aqueles que residam com tempo menor que seis meses nos bairros selecionados, por entender que podiam não estar bem familiarizados com essas comunidades.

A coleta de dados ocorreu nos bairros A, B e $\mathrm{C}$, em ruas e casas selecionadas aleatoriamente. Os participantes foram abordados em suas próprias casas. Não foi incluso mais de um membro de uma mesma casa e família, independente do grau de parentesco. Para a seleção deste participante na residência, considerou-se aquele que estava no momento da entrevista e o primeiro que preenchesse todos os critérios de inclusão. Caso a residência visitada estivesse fechada, não realizou tentativas posteriores, sendo escolhida outra residência de forma aleatória.

No bairro A foram abordadas 35 pessoas, sendo que 15 não aceitaram participar do estudo; no bairro B foram abordadas 26 pessoas e seis se recusaram; e no bairro C, 30 pessoas foram abordadas e 10 declinaram. A determinação do tamanho amostral nos três bairros baseou-se somente nos aspectos qualitativos, definido conforme saturação dos dados, até atingir o objetivo do estudo. Assim, a amostra final do estudo foi composta por 60 participantes.

As entrevistas tiveram duração média de $40 \mathrm{~min}$ e foram guiadas por um instrumento principal, contendo perguntas fechadas (aspectos sóciodemográficos e estilo de vida) e abertas (relativas às informaçóes sobre a proximidade e o custo-consumo de bebidas alcoólicas), elaborado pelos próprios pesquisadores. Este instrumento foi validado em teste piloto previamente com amostra semelhante à pesquisa nos bairros $\mathrm{A}, \mathrm{B}$ e $\mathrm{C}$, para possíveis adequações, porém não observou a necessidade de alteraçóes.

De forma complementar, para verificar o padrão do uso de álcool pelos participantes do estudo, utilizou-se o instrumento Alcohol Use Disorders Identification Test (AUDIT). Trata-se de um questionário desenvolvido em 1982 pela Organização Mundial de Saúde (OMS), para a avaliação do uso de álcool na população mundial. Compóe-se de 10 perguntas, cada questão tem uma margem de 0 a 4, possibilitando uma pontuação final de 0 a 40 pontos. Tem como resultado: baixo consumo (0 a 7 pontos) consumo de risco (8 a 15 pontos), padrão de uso nocivo (16-19 pontos) e diagnóstico de dependência (20-40 pontos).

Para análise dos dados qualitativos, utilizouse o Discurso do Sujeito Coletivo (DSC). Assim, seguiram-se as quatro etapas operacionais: seleção das expressôes-chave (ECH) de cada discurso, podendo ser contínuas ou descontínuas; identificação da ideia central (IC) de cada uma dessas ECH; identificação das IC semelhantes ou complementares; junção das ECH referentes às IC e por fim, formou-se uma síntese do discurso ${ }^{(17)}$.

Em relação aos dados quantitativos, procedeuse a digitação dupla, como forma de monitorar possíveis inconsistências ou erros. $\mathrm{Na}$ sequência, para avaliar as associaçóes entre as variáveis de custoconsumo (teor alcoólico, preço e compra) com o do padrão de uso de álcool ( $A U D I T)$, foi utilizado o programa BioEstat versão 5.0, por meio da análise de correlação "Coeficiente de Contingência C", onde $\mathrm{C}=0$ determina que não há associação entre as variáveis, quando $C \neq 0$ há correlação entre duas variáveis: $<0,1$ fraca; 0,1 a 0,3 moderada e $>0,3$ forte, sendo adotado o nível de significância de 5\% $(\mathrm{p}<0,05)$.

Foram respeitados todos os aspectos éticos em pesquisa, de acordo com a Resolução 466/12, iniciando o estudo somente após aprovação do Comitê de Ética em Pesquisa com Seres Humanos da Universidade do Estado de Mato Grosso (CEP/ UNEMAT) sob CAAE 17172919.0.0000.5166 e parecer 3.501.822. Todos os participantes do estudo assinaram o Termo de Consentimento Livre e Esclarecido (TCLE).

\section{RESULTADOS}

Integraram o estudo 60 participantes, sendo 30 mulheres (19 a 60 anos), com início do consumo de álcool entre 13 e 40 anos, e 30 homens (19 a 72 anos), com início do consumo de álcool entre 9 e 26 anos. Entre ambos, predominou-se o consumo de cerveja, adquirindo a bebida em mercados do próprio bairro (Tabela 1).

O Quadro 1a y 1 b apresenta as IC e DSC das participantes do estudo frente as narrativas sobre motivos de ingestáo de bebidas alcoólicas e meios para redução do consumo. Inclui também o perfil de uso quantitativo de possíveis influências do teor 
alcoólico e preço no consumo, além da aquisição de de baixo consumo (A e B). Em contrapartida, outras bebidas alcoólicas na ausência da principal. apontam que o preço da bebida é um fator que Prevalece mulheres com consumo de risco. O influencia seu consumo ( $\mathrm{A}, \mathrm{B}$ e C) e quando não há teor alcoólico é um aspecto que não interfere no sua bebida alcoólica de preferência adquirem outra consumo, especialmente em áreas com predomínio (A, B e C).

Tabela 1. Caracterização sociodemográfica dos participantes do estudo. Região da Amazônia Legal, Brasil (n=60).

\begin{tabular}{|c|c|c|c|c|c|}
\hline \multicolumn{2}{|c|}{ VARIÁVEIS } & \multicolumn{2}{|c|}{$\begin{array}{c}\text { Sexo feminino } \\
\quad(n=30)\end{array}$} & \multicolumn{2}{|c|}{$\begin{array}{c}\text { Sexo masculino } \\
(\mathrm{n}=30)\end{array}$} \\
\hline & & fr & $\%$ & fr & $\%$ \\
\hline \multirow{2}{*}{ Cor } & Branca & 25 & 83,3 & 7 & 23,3 \\
\hline & Não branca & 5 & 16,7 & 23 & 76,7 \\
\hline \multirow{2}{*}{ Escolaridade } & $<8$ anos & 25 & 83,3 & 20 & 66,7 \\
\hline & $>8$ anos & 5 & 16,7 & 10 & 33,3 \\
\hline \multirow{2}{*}{ Religião } & Católica & 27 & 90,0 & 25 & 83,3 \\
\hline & Não Católica & 3 & 10,0 & 5 & 16,7 \\
\hline \multirow{2}{*}{ Estado civil } & Solteiro & 16 & 53,3 & 18 & 60,0 \\
\hline & Casado & 14 & 46,7 & 12 & 40,0 \\
\hline \multirow{2}{*}{ Trabalho Remunerado } & Sim & 28 & 93,3 & 29 & 96,7 \\
\hline & Não & 2 & 6,7 & 1 & 3,30 \\
\hline \multirow{3}{*}{ Horas de sono } & $<4$ horas & - & - & 6 & 20,0 \\
\hline & 4 a 8 horas & 26 & 86,7 & 24 & 80,0 \\
\hline & $>8$ horas & 4 & 13,3 & - & - \\
\hline
\end{tabular}

fr= Frecuencia

Quadro 1a. Aspectos que implicam no custo-consumo de bebidas alcoólicas entre mulheres, Região da Amazônia Legal, Brasil, agosto a dezembro $2019(\mathrm{n}=30)$.

\begin{tabular}{|c|c|c|c|c|c|c|c|c|c|c|}
\hline \multirow{3}{*}{$\begin{array}{c}\text { Bairros } \\
\left(\mathrm{N}^{\circ} \text { participantes }\right)\end{array}$} & \multicolumn{4}{|c|}{ AUDIT } & \multirow{2}{*}{\multicolumn{2}{|c|}{$\begin{array}{c}\text { O teor } \\
\text { alcoólico } \\
\text { influencia seu } \\
\text { consumo? }\end{array}$}} & \multirow{2}{*}{\multicolumn{2}{|c|}{$\begin{array}{c}\text { O preço } \\
\text { da bebida } \\
\text { influencia seu } \\
\text { consumo? }\end{array}$}} & \multirow{2}{*}{\multicolumn{2}{|c|}{$\begin{array}{l}\text { Quando náo tem } \\
\text { a bebida alcoólica } \\
\text { que gosta, você } \\
\text { compra outra para } \\
\text { substituir? }\end{array}$}} \\
\hline & $\begin{array}{c}\text { Baixo } \\
\text { consumo }\end{array}$ & $\begin{array}{c}\text { Consumo } \\
\text { de risco }\end{array}$ & $\begin{array}{c}\text { Uso } \\
\text { nocivo }\end{array}$ & Dependência & & & & & & \\
\hline & $\mathrm{fr}$ & $\mathrm{fr}$ & $\mathrm{fr}$ & $\mathrm{fr}$ & Náo & Sim & Não & Sim & Náo & Sim \\
\hline Bairro A (10) & 4 & 4 & 2 & 0 & 6 & 0 & 0 & 8 & 0 & 9 \\
\hline Bairro B (10) & 6 & 3 & 0 & 1 & 9 & 0 & 0 & 9 & 0 & 9 \\
\hline Bairro C (10) & 1 & 6 & 3 & 0 & 0 & 7 & 0 & 10 & 0 & 10 \\
\hline
\end{tabular}


Quadro 1b. Aspectos que implicam no custo-consumo de bebidas alcoólicas entre mulheres, Região da Amazônia Legal, Brasil, agosto a dezembro $2019(\mathrm{n}=30)$.

\begin{tabular}{|c|c|c|}
\hline \multirow{2}{*}{$\begin{array}{c}\text { Bairros } \\
\left(\mathrm{N}^{\circ} \text { participantes }\right)\end{array}$} & \multicolumn{2}{|c|}{ Discurso do Sujeito Coletivo (DSC) } \\
\hline & Motivos para você ingerir bebidas alcoólicas? & $\begin{array}{l}\text { O que é necessário para reduzir seu } \\
\text { consumo? }\end{array}$ \\
\hline \multirow{4}{*}{ Bairro A (10) } & IC 1 - Socialização/ Interação & IC 1 - Obstinação \\
\hline & $\begin{array}{l}\text { Bebo mais por influência, quando estou na } \\
\text { companbia de amigos ou junto com minha } \\
\text { familia, para passar o tempo e interagir } \mathrm{ECH} \\
(\mathrm{fr}=6) \text {. }\end{array}$ & $\begin{array}{l}\text { Precisarei ter força de vontade, opiniäo } \\
\text { própria, vergonha na cara e ter noçâo dos } \\
\text { beneficios de uma vida sem precisar beber } \\
\text { para se sentir bem } \mathrm{ECH}(\mathrm{fr}=8) \text {. }\end{array}$ \\
\hline & IC 2 - Alívio e bem-estar & IC 2 - Suporte externo \\
\hline & $\begin{array}{l}\text { Bebo em situação de solidão, angústia ou } \\
\text { estresse. Bebendo me sinto aliviada e melhora } \\
\text { minha autoestima. Além disso, tenho sensação } \\
\text { de prazer e felicidade } \mathrm{ECH} \text { ( } \mathrm{fr}=4) \text {. }\end{array}$ & $\begin{array}{c}\text { Basta querer parar, mas precisarei do apoiol } \\
\text { suporte de pessoas e buscar ajuda em Deus } \\
\text { ECH }(\mathrm{fr}=2)\end{array}$ \\
\hline \multirow{6}{*}{ Bairro B (10) } & IC 1 - Diversão & IC 1 - Obstinaçáo \\
\hline & $\begin{array}{l}\text { Bebo para distrair, por diversão mesmo e para } \\
\text { acompanhar amigos. É também uma tradição } \\
\text { da minha família se reunir para confraternizar } \\
\text { e beber, como forma de diversáo. Nas festas, } \\
\text { beber é uma regra ECH }(\mathrm{fr}=8) \text {. }\end{array}$ & $\begin{array}{l}\text { Simplesmente querer parar, ter atitude, } \\
\text { opiniäo própria e ser persistente } \mathrm{ECH}(\mathrm{fr}=5) \text {. }\end{array}$ \\
\hline & IC 2 - Alívio e bem-estar & IC 2 - Afastar-se de pares que consomem \\
\hline & $\begin{array}{l}\text { Bebo para aliviar tensöes e relaxar. Me sinto } \\
\text { bem quando bebo } \mathrm{ECH}(\mathrm{fr}=2)\end{array}$ & $\begin{array}{l}\text { Só eu ficar mais dentro de casa, afastar um } \\
\text { pouco dos meus amigos e não andar com } \\
\text { quem possa me influenciar } \mathrm{ECH}(\mathrm{fr}=3) \text {. }\end{array}$ \\
\hline & & IC 3 - Evitar o acesso à bebida \\
\hline & & $\begin{array}{l}\text { Não comprar nenhum tipo de bebida } \\
\text { alcoólica, nem aquelas com teor menor, e } \\
\text { evitar qualquer forma de contato com o álcool } \\
\mathrm{ECH}(\mathrm{fr}=2) \text {. }\end{array}$ \\
\hline \multirow{8}{*}{ Bairro C (10) } & IC 1 - Socialização/ Interação & IC 1 - Obstinação \\
\hline & $\begin{array}{c}\text { Por vontade própria. É mais para alegrar, ficar } \\
\text { mais engraçada e leve, rir bastante. Me faz } \\
\text { sentir bem, principalmente para comemorar } \\
\text { quando estou alegre } \mathrm{ECH}(\mathrm{fr}=8) .\end{array}$ & $\begin{array}{c}\text { Ter opiniäo e sempre dizer näo. Ter consciência } \\
\text { do mal que pode acontecer se beber todos os } \\
\text { dias e colocar como meta parar de beber. Não } \\
\text { desistir diante de influências negativas. Se } \\
\text { controlar para não dar o primeiro gole } \mathrm{ECH} \\
(\mathrm{fr}=4) .\end{array}$ \\
\hline & IC 2 - Diversão & IC 2 - Afastar-se de pares que consomem \\
\hline & $\begin{array}{l}\text { Bebo por diversão, em momentos entre os } \\
\text { amigos e familia. Na minha família sempre } \\
\text { bebemos nas festanças } \mathrm{ECH}(\mathrm{fr}=2) \text {. }\end{array}$ & $\begin{array}{c}\text { Tenho que sair da roda de pessoas que bebem, } \\
\text { dizer não para aqueles amigos e ficar mais em } \\
\text { casa } \mathrm{ECH}(\mathrm{fr}=3) \text {. }\end{array}$ \\
\hline & & IC 3 - Evitar o acesso às bebidas \\
\hline & & $\begin{array}{c}\text { Evitar contato com a bebida, principalmente } \\
\text { em festas e idas em reuniöes com os amigos } \\
\mathrm{ECH}(\mathrm{fr}=2) .\end{array}$ \\
\hline & & IC 4 - Suporte externo \\
\hline & & $\begin{array}{l}\text { Ter muito esforço, com apoio de profissionais } \\
\text { ECH ( } \mathrm{fr}=1) .\end{array}$ \\
\hline
\end{tabular}

$\mathrm{fr}=\mathrm{N}^{\circ}$ participantes; IC: Ideas central; ECH: Expressóes-chave. 
Conforme os discursos, entre os motivos para ingerirem as bebidas alcoólicas, destaca-se a socialização/interação, principalmente em áreas que prevalecem o consumo de risco (A e C). Para reduzirem o consumo, elas apontam que é necessário, principalmente a obstinação (A, B e C) e se afastarem de pares que consomem (A e C).

No Quadro 2a y $2 \mathrm{~b}$ apresenta as IC e DSC dos participantes do estudo, em resposta aos mesmos questionamentos das mulheres. Em ambos os bairros investigados, os homens se mostram bastante envolvidos com o consumo de bebidas alcoólicas, em situação de dependência química (A e C), seguido de uso nocivo (A e B). O teor alcoólico é um fator que influencia o consumo, assim como está mais relacionado àqueles que consomem mais as bebidas ( $\mathrm{A}, \mathrm{B}$ e $\mathrm{C}$ ), seja como uso nocivo ( $\mathrm{A}$ e $\mathrm{B}$ ) ou na condição de dependência (A e C). Os preços das bebidas também foram apontados como fator que influencia o consumo (B e C). Diante da falta da bebida favorita, compram outra para substituir (A, B e C).
Conforme os discursos, entre os principais motivos para ingerirem as bebidas alcoólicas, destaca-se fatores relacionados ao alívio e bem-estar (A, B e C), e para fins de diversão (A e B). Para reduzirem o consumo, apontam que é necessário, principalmente a obstinação (A e B) e se afastarem de pares que consomem (A e C).

Em relação a totalidade dos participantes do estudo $(\mathrm{n}=60)$, verificou-se a associação das variáveis de custo-consumo com os resultados do AUDIT (Tabela 2).

$\mathrm{Na}$ Tabela 2, observa-se uma forte associação do teor alcoólico $(\mathrm{C}=0,481 ; \mathrm{p}=0,0004)$ e preço da bebida $(C=0,386 ; p=0,0197)$ com todos os tipos de padrão de uso de álcool identificado entre os participantes do estudo. Já a variável "compra outra bebida" teve moderada associaçáo (c=0,252; $\mathrm{p}=0,251)$, contudo não apresentou evidência estatística.

Quadro 2a. Aspectos que implicam no custo-consumo de bebidas alcoólicas entre homens, agosto a dezembro de 2019. Regiáo da Amazônia Legal, Brasil (n=30).

\begin{tabular}{|c|c|c|c|c|c|c|c|c|c|c|}
\hline \multirow{3}{*}{$\begin{array}{c}\text { Bairros } \\
\left(\mathrm{N}^{\circ} \text { participantes }\right)\end{array}$} & \multicolumn{4}{|c|}{ AUDIT } & \multirow{2}{*}{\multicolumn{2}{|c|}{$\begin{array}{c}\text { O teor } \\
\text { alcoólico } \\
\text { influencia seu } \\
\text { consumo? }\end{array}$}} & \multirow{2}{*}{\multicolumn{2}{|c|}{$\begin{array}{c}\text { O preço } \\
\text { da bebida } \\
\text { influencia seu } \\
\text { consumo? }\end{array}$}} & \multirow{2}{*}{\multicolumn{2}{|c|}{$\begin{array}{c}\text { Quando náo tem } \\
\text { a bebida alcoólica } \\
\text { que gosta, você } \\
\text { compra outra para } \\
\text { substituir? }\end{array}$}} \\
\hline & \multirow{2}{*}{$\begin{array}{c}\begin{array}{c}\text { Baixo } \\
\text { consumo }\end{array} \\
\mathrm{fr} \\
\end{array}$} & \multirow{2}{*}{$\begin{array}{c}\begin{array}{c}\text { Consumo } \\
\text { de risco }\end{array} \\
\mathrm{fr}\end{array}$} & \multirow{2}{*}{$\begin{array}{c}\begin{array}{c}\text { Uso } \\
\text { nocivo }\end{array} \\
\mathrm{fr} \\
\end{array}$} & \multirow{2}{*}{$\begin{array}{c}\text { Dependência } \\
\mathrm{fr} \\
\end{array}$} & & & & & & \\
\hline & & & & & Não & Sim & Não & Sim & Não & Sim \\
\hline Bairro A (10) & 2 & 0 & 4 & 4 & 0 & 8 & 8 & 0 & 0 & 7 \\
\hline Bairro B (10) & 2 & 2 & 4 & 2 & 0 & 8 & 0 & 8 & 0 & 10 \\
\hline Bairro C (10) & 0 & 4 & 0 & 6 & 0 & 9 & 0 & 10 & 0 & 10 \\
\hline
\end{tabular}

fr= Em AUDIT e Não o Sim= No respostas. 
Quadro 2b. Aspectos que implicam no custo-consumo de bebidas alcoólicas entre homens, agosto a dezembro de 2019. Regiáo da Amazônia Legal, Brasil ( $\mathrm{n}=30)$.

\begin{tabular}{|c|c|c|}
\hline \multirow{2}{*}{$\begin{array}{c}\text { Bairros } \\
\left(\mathrm{N}^{\circ} \text { participantes }\right)\end{array}$} & \multicolumn{2}{|c|}{ Discurso do Sujeito Coletivo (DSC) } \\
\hline & Motivos para você ingerir bebidas alcoólicas? & O que é necessário para reduzir seu consumo? \\
\hline \multirow{8}{*}{ Bairro A (10) } & IC 1 - Diversão & IC 1 - Obstinação \\
\hline & $\begin{array}{c}\text { Costumo beber em casa, para comemorar e } \\
\text { confraternizar na companbia da familia e amigos } \\
\mathrm{ECH}(\mathrm{fr}=2)\end{array}$ & $\begin{array}{l}\text { Ter determinaçäo, força de vontade, persistência e } \\
\text { não mais beber. É importante pensar e dizer, não } \\
\text { é isso que eu quero pra mim } \mathrm{ECH}(\mathrm{fr}=5) \text {. }\end{array}$ \\
\hline & IC 2 - Alívio e bem-estar & IC 2 - Afastar-se de pares que consomem \\
\hline & $\begin{array}{l}\text { Bebo para me sentir feliz, aliviar o cansaço, para } \\
\text { me sentir bem e esquecer os problemas. Bebo } \\
\text { também para alegrar o coração } \mathrm{ECH}(\mathrm{fr}=5) \text {. }\end{array}$ & $\begin{array}{l}\text { Näo ficar muito perto de pessoas que bebem. Os } \\
\text { amigos podem acabar influenciando e deixar } \\
\text { você com vontade de beber } \mathrm{ECH}(\mathrm{fr}=2) \text {. }\end{array}$ \\
\hline & IC 3 - Estimulação & IC 3 - Evitar o acesso às bebidas \\
\hline & $\begin{array}{l}\text { Por adrenalina, para poder ficar "ligadão", } \\
\text { quanto mais forte a bebida, melhor é o efeito } \\
\text { ECH }(\mathrm{fr}=2) \text {. }\end{array}$ & $\begin{array}{c}\text { Só ir morar em fazenda, ficar distante da cidade, } \\
\text { das festas e evitar passar perto de bar } \mathrm{ECH} \\
(\mathrm{fr}=2) \text {. }\end{array}$ \\
\hline & IC 4 - Influência dos amigos & IC 4 - Suporte religioso \\
\hline & $\begin{array}{c}\text { Acho que é mais por influência, a maioria de } \\
\text { meus amigos bebem } \mathrm{ECH}(\mathrm{fr}=1) \text {. }\end{array}$ & $\begin{array}{l}\text { Para parar de beber, só indo à igreja. A religiāo } \\
\text { pode controlar esse vício } \mathrm{ECH}(\mathrm{fr}=1) .\end{array}$ \\
\hline \multirow{6}{*}{ Bairro B (10) } & IC 1 - Diversão & IC 1 - Novo estilo de vida \\
\hline & $\begin{array}{l}\text { Bebo para me divertir e distrair, quando estou } \\
\text { com meus amigos e familiares } \mathrm{ECH}(\mathrm{fr}=5) \text {. }\end{array}$ & $\begin{array}{l}\text { Vou precisar fazer alguma coisa pra me distrair } \\
\text { pra ocupar minha mente e resistir. Buscar outras } \\
\text { formas de me divertir. Tenho que mudar minha } \\
\text { rotina, praticar esporte vai ajudar bastante me } \\
\text { distrair da vontade de beber } \mathrm{ECH}(\mathrm{fr}=6) \text {. }\end{array}$ \\
\hline & IC 2 - Alívio e bem-estar & IC 2 - Afastar-se de pares que consomem \\
\hline & $\begin{array}{l}\text { Bebo por vontade mesmo, o efeito do álcool é } \\
\text { bom para relaxar e aliviar a tensáo e canseira. } \\
\text { Quando bebo me sinto legal e bem confortável } \\
\mathrm{ECH}(\mathrm{fr}=5) \text {. }\end{array}$ & $\begin{array}{l}\text { Teria que mudar as minhas amizades e evitar } \\
\text { convivência com pessoas que bebem e seus } \\
\text { ambientes, isso influencia muito ECH (fr=4). }\end{array}$ \\
\hline & & IC 3 - Suporte religioso \\
\hline & & $\begin{array}{l}\text { Acho que a religiäo poderia me ajudar a parar de } \\
\text { beber } \mathrm{ECH}(\mathrm{fr}=1) \text {. }\end{array}$ \\
\hline \multirow{6}{*}{ Bairro C (10) } & IC 1 - Diversão & IC 2 - Obstinação \\
\hline & $\begin{array}{l}\text { Bebo para curtir e me divertir, em festas e datas } \\
\text { comemorativas com amigos e familia } \mathrm{ECH} \\
\qquad(\mathrm{fr}=5) .\end{array}$ & $\begin{array}{l}\text { Para parar de beber é preciso muita coragem e } \\
\text { opiniäo. A atitude é fundamental, ter iniciativa } \\
\text { e não aceitar a bebida quando alguém oferecer } \\
\text { ECH (fr=6). }\end{array}$ \\
\hline & IC 2 - Estimulaçáo & IC 1 - Afastar-se de pares que consomem \\
\hline & $\begin{array}{c}\text { Bebo pela adrenalina, gosto, pois, fico mais } \\
\text { animado, corajoso. E para alegrar a vida } \mathrm{ECH} \\
(\mathrm{fr}=3)\end{array}$ & $\begin{array}{l}\text { Preciso evitar estar com pessoas que bebem que } \\
\text { gostam de beber. Meus amigos sempre estäo me } \\
\text { chamando para beber, tenho que parar de andar } \\
\text { com eles } \mathrm{ECH}(\mathrm{fr}=4) \text {. }\end{array}$ \\
\hline & IC 3 - Acalento e conforto & \\
\hline & $\begin{array}{l}\text { Bebo para curar a solidão, ausência da família. } \\
\text { Serve também para aliviar e dar conforto a alma } \\
\text { ECH }(\mathrm{fr}=2) \text {. }\end{array}$ & \\
\hline
\end{tabular}

$\mathrm{fr}=\mathrm{N}^{\circ}$ participantes; IC: Ideas central; ECH: Expressóes-chave. 
Identificou-se que em todas as regióes (A, B e C) a dependência química esteve mais presente entre os homens do que as mulheres (12:1). Apesar de ter observado também que entre as mulheres há indicativos de progressão do consumo, elas se mantêm mais conscientes quanto aos danos, mesmo sob comportamento de risco. Essa característica não reflete somente uma tendência local, mas se assemelha ao comportamento nacional ${ }^{(18)}$ e a de outros países como nos Estados Unidos ${ }^{(19)}$, Suécia ${ }^{(20)}$ e Austrália ${ }^{(21)}$.

No Brasil, nos últimos anos, especificamente em relaçáo ao consumo feminino de cerveja houve um crescimento, enquanto em 2011 38\% a consumiam, em 2015 passou para $60 \%{ }^{(22)}$. Esse cenário ainda se agrava, pois, as mulheres enfrentam julgamentos quer seja em beber ${ }^{(23)}$ como em recusar a bebida ${ }^{(24)}$, contradiçôes que ao invés de auxiliar no processo decisório sensato frente ao consumo de álcool, as distanciam dos serviços de saúde e apoio profissional ${ }^{(25)}$, ampliando os motivos para aderir ou intensificar o consumo, bem como o desconhecimento sobre a gravidade da adição.

Em relação ao custo-consumo de álcool entre as mulheres, pesquisa conduzida em Portugal, verificou que no instante da compra da bebida alcoólica, elas tendem a apresentar dificuldades em diferenciar os diversos tipos disponíveis no mercado, atentando-se exclusivamente ao valor ${ }^{(26)}$, o que possivelmente justifica a aquisição de outro tipo de bebida, quando náo encontram sua preferida, como apontado no presente estudo.

Em Dourados (MS), estudo indicou que o consumo feminino está relacionado com acesso facilitado à diversidade de bebidas encontradas a preços relativamente baixos, corroborando assim com a premissa de que a oferta é capaz de gerar demanda ${ }^{(27)}$. Além disso, as mulheres parecem, por aspectos sociais, não assumirem naturalmente a compra de bebidas e preferem referir baixa frequência no consumo de álcool. Entretanto, estudo realizado em Recife (PE) indica que mesmo aquelas que possuíam baixo consumo, nas ocasióes em que bebiam costumavam consumir dez ou mais doses, tornando mais vulnerável e em risco de sofrer os impactos do álcool, dada a menor tolerância que possuem quando comparado aos homens ${ }^{(28)}$.

O teor alcoólico não foi representativo entre 
a maioria das participantes deste estudo (A e B). Segundo algumas pesquisadoras, o abandono do consumo de bebidas de menor teor alcoólico para graduaçóes maiores ocorre quase exclusivamente na condição de migração do padrão de uso de álcool, em direção à dependência química ${ }^{(23)}$, diferentemente dos achados do estudo, em que as mulheres se concentraram entre consumo de risco (A e C) e baixo consumo (A e B).

Mesmo tendo identificado quantitativo correspondente entre preço e consumo ( $\mathrm{A}, \mathrm{B}$ e $\mathrm{C}$ ), as participantes afirmam que normalmente bebem de forma compartilhada com homens nas festividades, assim, na maioria das vezes, não possuem nenhum custo para consumir a bebida. Segundo algumas pesquisas, esses aspectos do custo-consumo entre as mulheres ainda são influenciados, quando há conhecimento sobre a bebida, os tipos de padróes de consumo ${ }^{(29)}$ e/ou quando o consumo ocorre em contexto familiar ${ }^{(30,31)}$.

No grupo dos homens, entre os aspectos que implicam no custo-consumo, o teor alcoólico (A, B e C) e o preço ( $\mathrm{B}$ e C) se corresponderam. Algumas evidências científicas confirmam esses achados, ao revelarem que o consumo de bebidas alcoólicas faz parte do processo de socializaçáo do homem, logo consomem por questóes culturais e de gênero ${ }^{(32)}$. Normalmente optam por bebidas de menor teor alcoólico e com maior aceitação coletiva, como a cerveja, o que os levam a ingerir em maior quantidade ${ }^{(33)}$, tendo como balizador o preço e a disponibilidade do produto $^{(34)}$.

Paralelamente, sabe-se que frequentar ambientes de bares e casas noturnas estimula o consumo de bebidas de maior teor alcoólico, como os destilados ${ }^{(35)}$, porém esse consumo não foi observado no presente estudo. Um dos motivos para essa distinção, talvez seja, porque a maioria (70\%) dos homens (A, B e C) bebe com frequência somente no ambiente doméstico, sustentando o hábito popular de consumo de cerveja.

Estudo realizado em Washington (EUA), não verificou diferenças significativas entre os diversos padróes de consumo quanto aos custos de compra, tamanho da garrafa ou tipo de estabelecimento escolhido, porém constatou que usuários em situação de dependência química escolhem bebida com preço menor, maior conteúdo $(\mathrm{ml})$ e em comércios mais próximo de suas residências ${ }^{(16)}$. Esses aspectos corroboram com os achados do estudo em relação aos aspectos de custo-consumo investigados e na constatação que os participantes adquirem suas bebidas em estabelecimentos do próprio bairro onde residem (A, B e C).

Por outro lado, na África entre as políticas que envolvem o custo-consumo, observa-se o apoio ao aumento da idade mínima para beber, ao invés do aumento dos $\operatorname{preços}^{(36)}$. Essa concepçáo pode corresponder a mesma dos participantes do presente estudo, pelo fato de terem iniciado o consumo de álcool com uma idade tenra e por apontar estratégias somente para outros e náo para si, uma vez que, a alteração de preços, tende a impactar diretamente no seu consumo.

$\mathrm{Na}$ Escócia, após a implantação do preço unitário mínimo nas compras de álcool houve uma redução nas compras semanais de $9,5 \mathrm{~g}$ de álcool por adulto por família, chegando até a redução de $15 \mathrm{~g}$, especificamente entre famílias de baixa renda ${ }^{(37)}$. Para tanto, pesquisadores russos revelam que quaisquer políticas de redução do consumo devem incluir outras medidas, como por exemplo, restriçóes ao número de pontos de venda ou seus horários de abertura ${ }^{(38)}$. Essa perspectiva, também foi confirmada em estudo realizado na Estônia, apontando que ainda que o preço do álcool seja apenas uma ferramenta da política de redução do consumo, há necessidade de maior atenção à disponibilidade do álcool, na compreensão que as restriçóes físicas e econômicas dessas bebidas resultem em melhorias e menos ônus para a saúde pública $^{(39)}$.

Nos discursos das mulheres, percebeu-se que o consumo ocorre como meio de/para socialização, enquanto entre os homens servem para aliviar as tensóes e encontrar bem-estar. No entanto, ambos são convictos que a melhor maneira de reduzir seu consumo é a obstinação. Estudo realizado em Vitória da Conquista (BA) com pessoas de 20 a 59 anos, de ambos os sexos, mas predominantemente mulheres, revelou que o processo de socialização entre amigos tem sido o fator que mais colabora no aumento do consumo de bebidas alcoólicas ${ }^{(40)}$. Mesmo sob essa influência e necessidade de afastamento desses bebedores, pesquisador americano aponta que tanto as mulheres como os homens que consomem álcool em excesso, podem ter esse hábito controlado e cessado definitivamente, com obstinação sem necessariamente receber um tratamento específico $^{(41)}$. Apesar disso, o aconselhamento, 
apoio e acompanhamento profissional, torna-se um recurso indispensável para esse processo de recuperação ${ }^{(42)}$.

Ainda que neste estudo não tenha avaliado comorbidades associadas ao consumo, pesquisa na Suécia com pacientes em tratamento de artrite reumatoide verificou que o ato de deixar o consumo de álcool esteve relacionado principalmente com a preocupação com a doença. E o apoio familiar e suporte espiritual foram determinantes para alcançar resultados positivos ${ }^{(43)}$, semelhante aos achados da pesquisa.

Apesar das singularidades entre os gêneros participantes desse estudo, ao observar toda a amostra $(n=60)$, entre os aspectos de custoconsumo, houve forte associação do teor alcoólico e preço da bebida com todos os tipos de padrão de uso de álcool (AUDIT). Outro estudo menciona essas influências, e aponta também que embora no Brasil se mantenha o consumo de cervejas com teor alcoólico de 3,5 a 5\%, a procura por bebidas com menor teor/sem álcool têm crescido, seja em função de preocupaçóes relacionadas à saúde, respeito a legislação (Lei Seca) ou pela conscientização frente ao custo-benefício ${ }^{(44)}$.

Semelhante aos achados do estudo, pesquisa nos Estados Unidos com ambos os sexos, verificou que procurar cerveja esteve associado positivamente ao uso recente (>1 vez na última semana) e igualmente àqueles que em uma ocasião possuíam um consumo de alto volume. Entretanto, as associaçóes dos aspectos que determinam o custo-consumo também diferem no contexto da exposição a forças diferentes (exercidas/compreendidas) das políticas de álcool sobre a pessoa e coletividades ${ }^{(45)}$.

Essa complexidade na compreensão da dimensão do custo-consumo tanto nacionalmente como internacionalmente também reflete nos desafios colocados aos profissionais no enfrentamento e gestão do cuidado, quer seja na definição de estratégias de prevenção como no estabelecimento do cuidado terapêutico. As experiências exitosas para redução do consumo, como apontado nos estudos já citados, ressaltam que há mecanismos efetivos para interferir e mediar, mas requer um trabalho interdisciplinar e motivação/consciência coletiva, pois os aspectos que determinam o custo-consumo, premissa para fundamentar o planejamento das açóes na comunidade, transitam entre os eixos políticos, econômicos, sociais e de saúde.
Nesse ínterim, outro obstáculo vislumbrado é a concepção desse perfil de participantes do estudo, que sabem dos malefícios, conhecem pessoas que foram prejudicadas pelo consumo excessivo de álcool, porém não desejam reduzir ou cessar o consumo. Isso revela um processo de adormecimento do cuidado com a própria saúde, quer seja pela pouca proximidade e atenção dos profissionais frente às fontes influenciadores e modos de vida como pela maior tendência desse grupo em se tornar dependentes químicos, mantendo ou priorizando o consumo em detrimento de iniciativas para a busca por cuidado e recuperaçáo. Assim, preocupaçóes com os aspectos que compóem o custo-consumo e que são importantes elementos para engendrar projetos terapêuticos, são relevadas, talvez porque o alcoolismo não integra a agenda de compromissos dos profissionais, como os que atuam na atenção básica; ou esses aspectos ainda são entendidos pela maioria da sociedade como fatores que não implicam na adicção e em suas consequências.

Este estudo apresentou algumas limitaçôes, destacando a inclusão em uma mesma amostra de grupos com períodos distintos de início e intensificação do consumo. Todavia, sinalizou características e evidências ainda desconhecidas, para a instrumentalização de gestores e profissionais. Novos estudos devem ser implementados, a fim de fornecer subsídios para o cuidado desse perfil populacional, que ainda se esbarra em dificuldades no acesso a serviços de saúde que consigam atender suas demandas ligadas ao consumo de álcool.

\section{CONCLUSÃO}

No estudo, predominou mulheres com consumo de risco e homens na condição de dependência química. Para todos os homens, o teor alcoólico influencia o consumo, assim como o preço das bebidas. Entre as mulheres, sendo distinto somente o teor alcoólico, como aspecto que não interfere no custo-consumo. Entre ambos os gêneros, a falta da bebida favorita não é um impeditivo para absterem do consumo de álcool. E, embora apontem que uma das principais estratégias para redução do consumo seja a obstinaçáo, poucos fazem ou se motivam para isso.

Esses achados podem ser utilizados para fundamentar novas políticas públicas junto a essa 
população, em busca de maior suporte social e compreensáo dos usuários, familiares e comunidade sobre o viver em sobriedade e em relação a estilos de vida mais saudáveis. Para isso, é importante que os profissionais dos diversos dispositivos sociais e de saúde incluam em suas atividades planejadas

\section{REFERÊNCIAS}

1. Rehm J, lachenmeier DW, Room R. Why does society accept a higher risk for alcohol than for other voluntary or involuntary risks? BMC Medicine [Internet]. 2014 [citado 2021 fev 18]; 12: 189. Disponível em: https://bmcmedicine. biomedcentral.com/articles/10.1186/s12916-0140189-z

2. Monteiro MG. Alcohol y Salud Pública en América Latina: ¿cómo impedir un desastre sanitario? Adicciones [Internet]. 2013 [citado 2021 fev 18]; 25(2): 99-105. Disponível en: https:// www.adicciones.es/index.php/adicciones/article/ view/56/55

3. Blecher E, Liber A, Walbeek CV, Rossouw L. An international analysis of the price and affordability of beer. PLoS One [Internet]. 2018 [citado 2021 fev 18]; 13(12): e0208831. Disponível em: https:// journals.plos.org/plosone/article?id=10.1371/ journal.pone.0208831

4. Siegfried N, Parry C. Do alcohol control policies work? An umbrella review and quality assessment of systematic reviews of alcohol control interventions (2006 -2017). PLoS One [Internet]. 2019 [citado 2021 fev 18]; 14(4): e0214865. Disponível em: https://pubmed.ncbi.nlm.nih.gov/30969992/

5. Duailibi S, Laranjeira R. Políticas públicas relacionadas às bebidas alcoólicas. Rev Saúde Pública [Internet]. 2007 [citado 2021 fev 18]; 41(5): 839-848. Disponível em: https://doi. org/10.1590/S0034-89102007000500019

6. Blake MR, Lancsar E, Peeters A, Backholer K. The effect of sugar-sweetened beverage price increases and educational messages on beverage purchasing behavior among adults. Appetite [Internet]. 2018 [citado 2021 fev 18]; 126: 156-162. Disponível em: https://pubmed.ncbi.nlm.nih.gov/29627346/

7. Calcott P. Minimum unit prices for alcohol. J Health Econ [Internet]. 2019 [citado $2021 \mathrm{fev}$ 18]; 66: 18-26. Disponível em: https://pubmed.ncbi. nlm.nih.gov/31078015/

8. Wilsnack RW, Wilsnack SC, Gmel G, Kantor LW. Gender Differences in Binge Drinking. Alcohol e de rotina intervençóes sobre álcool e outras drogas, na perspectiva que haja o reconhecimento de espaço na própria comunidade para o diálogo, acolhimento, cuidado e gerenciamento de necessidades apresentadas.

Res [Internet]. 2018 [citado $2021 \mathrm{fev}$ 18]; 39(1): 57-76. Disponível em: https://www.ncbi.nlm.nih. gov/pmc/articles/PMC6104960

9. Patterson C, Emslie C, Mason O, Fergie G, Hilton $\mathrm{S}$. Content analysis of UK newspaper and online news representations of women's and men's 'binge' drinking: a challenge for communicating evidencebased messages about single-episodic drinking? BMJ Open [Internet]. 2016 [citado $2021 \mathrm{fev}$ 18]; 6(12): e013124. Disponível em: https://bmjopen. bmj.com/content/6/12/e013124

10. Oliveira AJ, Andrade FFF, Santos GMB, Ferro LRM, Rezende MM. As repercussóes do estigma social na busca de mulheres alcoolistas por tratamento especializado. Rev Uniandrade [Internet]. 2019 [citado $2021 \mathrm{fev}$ 18]; 20(3): 148158. Disponível em: https://revista.uniandrade.br/ index.php/revistauniandrade/article/view/1440

11. Marques MV, Silva Júnior DN, Santos EGO, Santos SSAN, Neves SMB, Amador AE. Distribuição espacial das mortes atribuíveis ao uso de álcool no Brasil. J Health Bio Sci [Internet]. 2020 [citado 2021 fev 18]; 8(1): 1-11. Disponível em: https:// periodicos.unichristus.edu.br/jhbs/article/view/ 2934

12. Nascimento VF, Lima CAS, Hattori TY, Terças ACP, Lemes AG, Luis MAV. Daily life of women with alcoholic companions and the provided care. An acad bras ciênc [Internet]. 2019 [citado 2021 fev 18]; 91(1): e20180008. Disponível em: https:// doi.org/10.1590/0001-3765201920180008

13. Nascimento VF, Borges JS, Cabral JF, TerçasTrettel ACP, Hattori TY, Lemes, AG, et al. Acesso a informaçôes sobre substâncias psicoativas e o consumo por agentes prisionais. Enferm Actual Costa Rica [Internet]. 2019 [citado $2021 \mathrm{fev}$ 18]; 36: 1-18. Disponível em: http://dx.doi. org/10.15517/revenf.v0i36.34918

14. Moretti-Pires RO, Corradi-Webster CM, Furtado EF. Consumo de álcool e atenção primária no interior da Amazônia: sobre a formação de médicos e enfermeiros para assistência integral. Rev Bras Educ Med [Internet]. 2011 [citado $2021 \mathrm{fev} \mathrm{18];}$ 35(2): 219-228. Disponível em: https://dx.doi. 
org/10.1590/S0100-55022011000200011

15. Kerr WC, Ye Y, Greenfield TK. Spirits purchasing and marijuana use behaviors of risky drinkers in the state of Washington from 2014 to 2016. Drug Alcohol Depend [Internet]. 2019 [citado $2021 \mathrm{fev}$ 18]; (198): 7-12. Disponível em: https://pubmed. ncbi.nlm.nih.gov/30856371/

16. Mendonça FD, Rocha SS, Pinheiro DLP, Oliveira SV. Região Norte do Brasil e a pandemia de COVID-19: análise socioeconômica e epidemiológica. J Health NPEPS [Internet]. 2020 [citado 2021 fev 18]; 5(1): 20-37. Disponível em: https:// periodicos.unemat.br/index.php/jhnpeps/article/ view/4535

17. Lefreve F, Lefreve AMC. O sujeito coletivo que fala. Interface [Internet]. 2006 [citado $2021 \mathrm{fev}$ 18]; 10(20): 517-24. Disponível em: https://doi. org/10.1590/S1414-32832006000200017

18. Sandoval GA, Monteiro MG, De Pinho CK, Shield K, Marinho F. Sociodemographics, lifestyle factors and health status indicators associated with alcohol consumption and related behaviours: a Brazilian population-based analysis. Public Health [Internet]. 2020 [citado $2021 \mathrm{fev}$ 18]; 178: 4961. Disponível em: https://pubmed.ncbi.nlm.nih. gov/31614326/

19. Tan $\mathrm{CH}$, Denny $\mathrm{CH}$, Cheal NE, Sniezek JE, Kanny D. Alcohol Use and Binge Drinking Among Women of Childbearing Age - United States, 2011-2013. Morb mortal wkly rep [Internet]. 2015 [citado $2021 \mathrm{fev}$ 18]; 64(37): 1042-1046. Disponível em: https://www.cdc.gov/ncbddd/fasd/ features/drinking-childbearing-age.html

20. Hunsberger M, Mehlig K, Björkelund C, Lissner L. Regular versus episodic drinking in Swedish women: reporting of regular drinking may be less biased by social desirability. Alcohol [Internet]. 2020 [citado $2021 \mathrm{fev}$ 18]; 86: 57-63. Disponível em: https://europepmc.org/article/med/31926927

21. Armstrong KA, Davey JD, Freeman JE, Young SJ. A qualitative exploration of apprehended women's experience of drink driving events. Transp res Part F Traffic psychol behav [Internet]. 2020 [citado 2021 fev 18]; 69: 49-60. Disponível em: https:// www.sciencedirect.com/science/article/abs/pii/ S1369847819304966

22. Pimenta LS. Comportamento dos consumidores de cerveja: escalonando os atributos influenciadores na decisão de compra dos produtos artesanais e industrializados. Rev FACTHUS [Internet]. 2019 [citado 2021 fev 18]; 1(2): 20-38. Disponível em: http://publicacoes.facthus.edu.br/index.php/ administracao/article/view/248

23. Silva MGB, Lyra TM. O beber feminino: socialização e solidão. Saúde debate [Internet].
2015 [citado 2021 fev 18]; 39(106): 772-781. Disponível em: https://doi.org/10.1590/0103-110 4201510600030017

24. Santos EL, Opaleye ES, Noto AR. Álcool e relaçôes de gênero: motivaçóes e vulnerabilidades na percepção de adolescentes. Psicol Estud [Internet]. 2018 [citado $2021 \mathrm{fev}$ 18]; 23: e40636. Disponível em: http://periodicos.uem.br/ojs/index.php/Psicol Estud/article/view/40636/pdf

25. Campelo A, Aguiar DM. Dependência química: direito ao tratamento e às intervençóes terapêuticas. Rev Nova Hileia [Internet]. 2017 [citado $2021 \mathrm{fev}$ 18]; 2(3): 1-15. Disponível em: http://periodicos. uea.edu.br/index.php/novahileia/article/view/1251

26. Marques CP, Guia ATB. A influência do gênero e da ocasião na frequência de consumo de vinho. TMStudies [Internet]. 2015 [citado $2021 \mathrm{fev}$ 18]; 11(2): 226-233. Disponível em: http://dx.doi. org/10.18089/tms.2015.11228

27. Estevão FQOL. Uso de álcool e fatores associados entre mulheres adultas da cidade de Dourados, MS [Tesis]. Repositório Mestrado em Ciências da Saúde UFGD [Internet], 2018 [citado $2021 \mathrm{fev}$ 18]; 1-50. Disponível em: http://repositorio.ufgd. edu.br/jspui/handle/prefix/1077

28. Silva MGB, Lyra TM, Diniz GT. O padrão de consumo de álcool entre as usuárias das Unidades de Saúde da Família no município do Recife (PE). Saúde debate [Internet]. 2019 [citado $2021 \mathrm{fev}$ 18]; 43(122): 836-847. Disponível em: https://doi. org/10.1590/0103-1104201912214

29. Islam MM, Hoffmann MW, Rahman MB Knowledge of low-risk drinking and its relationship with a reduction in alcohol consumption: Results from six waves of an Australian national survey. Addictive Behav [Internet]. 2019 [citado $2021 \mathrm{fev}$ 18]; 95: 172-177. Disponível em: https://pubmed. ncbi.nlm.nih.gov/30928662/

30. Iurkiv AAB. Impactos da dependência do álcool na vida social e familiar da mulher: uma visão humanista. Fac Sant'Ana rev [Internet]. 2019 [citado 2021 fev 18]; 2: 142-157. Disponível em: https://www.iessa.edu.br/revista/index.php/fsr/ article/view/621

31. Noel NE, Daniels KA, Ogle RL, Maisto SA, Lee AJR, Ehlke SJ, et al. Women's drinking decisions in sexually risky situations: Effects of a low level of intoxication. Addictive Behav [Internet]. 2015 [citado 2021 fev 18]; 47: 61-65. Disponível em: https://pubmed.ncbi.nlm.nih.gov/25879711/

32. Rosa LFA, Nascimento ARA. Representaçōes sociais de bebida alcoólica para homens universitários. Arq bras psicol [Internet]. 2015 [citado $2021 \mathrm{fev} \mathrm{18];}$ 67(1): 3-19. Disponível em: https://www.redalyc. org/articulo.oa?id=229039192002 
33. Fiala J, Sochor O, Klimusová H, Homolka M. Alcohol Consumption in Population Aged 25-65 Years Living in the Metropolis of South Moravia, Czech Republic. Cent euro j public health [Internet]. 2017 [citado $2021 \mathrm{fev}$ 18]; 25(3): 191199. Disponível em: https://pubmed.ncbi.nlm. nih.gov/29022677/

34. Monteiro MG. Políticas públicas para a prevenção dos danos relacionados ao consumo de álcool. Epidemiol Serv saúde [Internet]. 2016 [citado 2021 fev 18]; 25(1): 171-174. Disponível em: https:// doi.org/10.5123/S1679-49742016000100017

35. Carmo DRP, Faria FL, Terra MG, Santos MA, Pilon SC. Motivaçóes atribuídas por adultos ao consumo de bebidas alcoólicas no contexto social. Psicol teor prat [Internet]. 2018 [citado $2021 \mathrm{fev}$ 18]; 20(2): 225-239. Disponível em: http://pepsic. bvsalud.org/pdf/ptp/v20n2/pt_v20n2a09.pdf

36. Parry CDH, Trangenstein P, Lombard C, Jernigan DH, Morojele NK. Support for alcohol policies from drinkers in the City of Tshwane, South Africa: Data from the International Alcohol Control study. Drug Alcohol Rev [Internet]. 2018 [citado 2021 fev 18]; 37(Suppl 1): S210-S217. Disponível em: https://pubmed.ncbi.nlm.nih.gov/28493419/

37. O'Donnell A, Anderson P, Jané-Llopis E, Manthey J, Kaner E, Rehm J. Immediate impact of minimum unit pricing on alcohol purchases in Scotland: controlled interrupted time series analysis for 2015-18. BMJ [Internet]. 2019 [citado $2021 \mathrm{fev}$ 18]; 366: 15274. Disponível em: https://www.bmj. com/content/366/bmj.15274

38. Goryakin Y, Roberts B, Mckee M. Price elasticities of alcohol demand: evidence from Russia. Euro $\mathrm{j}$ health econ [Internet]. 2015 [citado $2021 \mathrm{fev}$ 18]; 16(2): 185-199. Disponível em: https://pubmed. ncbi.nlm.nih.gov/24535047/

39. Parna K. Alcohol consumption and alcohol policy in Estonia 2000-2017 in the context of Baltic and Nordic countries. Drug Alcohol Ver [Internet].
2019 [citado 2021 fev 18]; 1-7. Disponível em: https://onlinelibrary.wiley.com/doi/full/10.1111/ dar. 13008

40. Soares FJ, Oliveira DC, Oliveira PR, Lima TS, Alves ALR, Silva ML, et al. Análise dos motivos dos jovens e adultos consumirem álcool. Id on Line Rev Multidiscip Psicol [Internet]. 2017 [citado 2021 fev 18]; 11(35): 554-566. Disponível em: https:// idonline.emnuvens.com.br/id/article/view/756

41. Bishop FM. Self-guided Change: The most common form of long-term, maintained health behavior change. Health Psychol Open [Internet]. 2018 [citado $2021 \mathrm{fev} 18$ ]; 5(1): 2055102917751576. Disponível em: https://pubmed.ncbi.nlm.nih.gov/ 29375888/

42. Beard E, Brown J, Kaner E, West R, Michie S. Predictors of and reasons for attempts to reduce alcohol intake: A population survey of adults in England. PLoS One [Internet]. 2017 [citado 2021 fev 18]; 12(3): e0173458. Disponível em: https:// journals.plos.org/plosone/article?id=10.1371/ journal.pone. 0173458

43. Larsson I, Andersson MLE, Barfot SGroup. Reasons to stop drinking alcohol among patients with rheumatoid arthritis in Sweden: a mixedmethods study. BMJ Open [Internet]. 2018 [citado 2021 fev 18]; 8(12): e024367. Disponível em: https://pubmed.ncbi.nlm.nih.gov/30552279/

44. Valente Júnior AS, Alves FCD. Bebidas alcoólicas: cerveja. Cad Set Etene [Internet]. 2016 [citado 2021 fev 18]; 1(2): 10-17. Disponível em: https:// www.bnb.gov.br/documents/80223/1138347/2_ cerveja.pdf

45. Weitzman ER, Magane KM, Chen PH, Amiri H, Naimi TS, Wisk LE. Online Searching and Social Media to Detect Alcohol Use Risk at Population Scale. Am j prev med [Internet]. 2020 [citado 2021 fev 18]; 58(1): 79-88. Disponível em: https://www. ajpmonline.org/article/S0749-3797(19)30382-4/ fulltext 